\title{
Lusioersily
}

\section{Cognitive Sensor Networks: Towards Self-Adapting Ambient Intelligence for Pervasive Healthcare}

Baumgarten, M., \& Mulvenna, M. (2011). Cognitive Sensor Networks: Towards Self-Adapting Ambient Intelligence for Pervasive Healthcare. In Unknown Host Publication (pp. 366-369). IEEE.

Link to publication record in Ulster University Research Portal

Published in:

Unknown Host Publication

Publication Status:

Published (in print/issue): 01/01/2011

\section{Document Version}

Publisher's PDF, also known as Version of record

\section{General rights}

Copyright for the publications made accessible via Ulster University's Research Portal is retained by the author(s) and / or other copyright owners and it is a condition of accessing these publications that users recognise and abide by the legal requirements associated with these rights.

\section{Take down policy}

The Research Portal is Ulster University's institutional repository that provides access to Ulster's research outputs. Every effort has been made to ensure that content in the Research Portal does not infringe any person's rights, or applicable UK laws. If you discover content in the Research Portal that you believe breaches copyright or violates any law, please contact pure-support@ulster.ac.uk. 


\title{
Cognitive Sensor Networks:
}

\section{Towards Self-Adapting Ambient Intelligence for Pervasive Healthcare}

\author{
Matthias Baumgarten, Maurice Mulvenna \\ TRAIL Living Lab, School of Computing and Mathematics \\ University of Ulster \\ Belfast, United Kingdom \\ \{m.baumgarten, md.mulvenna)@ulster.ac.uk
}

\begin{abstract}
The importance as well as the availability of sensor-based technology and environments is constantly increasing. This is not only based on the latest advances in sensor technology but also due to the incorporation of more powerful communication and processing mechanisms into the sensor nodes which may, eventually, allow for the emergence of cognitive intelligence from within such networks themselves. Such platforms are collectively referred to as wireless sensor networks, which provide not only the capabilities to sense and to process information but also to act upon them, which allows to actively influence the underlying context. Consequently, future intelligent infrastructures will be largely based on sensor network technology that will provide the layers for contextual information gathering, knowledge processing as well as for adaptation and optimization mechanisms. This will pave the way for new types of application and services in the area of pervasive computing in healthcare and beyond that are fully autonomous in all aspects of their operations as well as their set-up and maintenance. This paper sketches the potential and challenges of future sensor networks that provide the foundations for self-adapting ambient intelligence and briefly discusses some of the requirements thereof.
\end{abstract}

Keywords: Future Sensor Networks, Intelligent Environments, Cognitive Services, Pervasive Healthcare

\section{INTRODUCTION}

Intelligent, sensor based devices and applications permeate the environments we live in at an unprecedented scale. They are becoming progressively intertwined with various activities of daily live and provide accurate and detailed information about the environment, the activities and interactions therein and also about the actors that exist within such environments. Sensor based information reflect important knowledge which can be modeled to reflect simple environment specific attributes, complex situations and behavior as well as personal or health related information such as user preferences or individual vital signs, respectively. Applications in the area of pervasive healthcare range from small scale, highly specific body area sensor networks such as discussed in [1], which reflect specialized micro-environments; over well known macro-environments used for example, in activity recognition as discussed in [2] and [3]; to large scale and often fully unsupervised pervasive environments in which a multitude of sensors are deployed. The latter in particular is aimed at introducing a level of ambient intelligence that will allow future intelligent infrastructures to become fully contextual aware and responsive to the benefit of its users. Applications of interest include but are not limited to the monitoring of physiological properties such as heart rate or blood pressure, the recognition and assistance of activities of daily life, the supervision of ambient environments in dependence of specific user characteristics, the provision of care in general, and the promotion and supervision of social activities and relations. In short the goal of future ambient environments in pervasive healthcare is to provide the required infrastructure to gather and model relevant aspects of daily life activities and to assist in the performance thereof also providing appropriate safety mechanisms in case of emergencies.

However, an important aspect in this context is the ability to understand and to adapt to new, previously unknown, conditions, which would constitute a certain degree of cognitive intelligence. Such intelligence would require (a) to sense current conditions, (b) to analyze them and eventually (c) to decide if and how to react to them. For such cognitive environments to become a reality it is no longer sufficient to use isolated sensor information or sequestered sensor infrastructures. Instead, it is necessary to link together all components available within a given infrastructure into a coherent self-adapting model of ambient intelligence from which cognitive capabilities can emerge that are based on the components involved and the interactions thereof. This becomes even more apparent when considering the large number of stakeholders involved in pervasive environments and the potentially infinitive number of volatile relations between them. In order to overcome this problem, future sensor networks need to be intrinsically interwoven with flexible reasoning mechanisms so that they are able to constantly establish, remove or refine the relations between all stakeholders on an operational as well as a social and business level. This will provide the knowledge base for advanced decision-making and prediction capabilities that would allow sensor networks to constantly self-adapt based on the dynamic context of the environment, individual stakeholders and, even more compelling the interactions and relations between them. Consequently, such environments would be more flexible with respect to their use, they would be more resilient and failsafe and would in general be able to provide a higher degree of interaction as well as understanding.

This paper is organized as follows. Section II outlines the potential of future intelligent and cognitive-aware sensor based environments, whereas Section III discusses some the 
requirements to be addressed for them to become a reality. Related work is discussed throughout whereas conclusions and future work are given in Section IV.

\section{Potential AND CHALlENGES FOR Cognitive SENSOR NETWORKS}

Among others, there are two main driving factors in the area of pervasive healthcare that require more intelligent cognitive enabled environments. Firstly, home based care services that enable the provision of effective care within the home environment thus avoiding early hospitalization, which would be more cost intensive. Secondly, ambient assistive living technologies (AAL) that specifically support the needs of the elderly and the disabled by providing mechanisms that enable them to live independently for longer within their home environment. This latter aspect is particular relevant considering that the average age of the populations of most countries is constantly increasing. It is projected that by 2040 , $14 \%$ of the world population will be reflected by older people (www.transgenerational.org). Intelligent environments can, in both cases, provide the means to monitor, analyze and react to individual contexts thus providing general safety layers in case of emergencies; optimized on-demand care services; assistance and guidance in the execution of certain activities; personalized health plans and services; social support and connectivity; purpose based stimuli promoting mental and physical wellbeing, and others.

Nevertheless, current technology for pervasive healthcare is often characterized to be highly specific in nature, purpose-build and, for proprietary reasons, often available as so called 'closed' systems which are not able to communicate with other components in support of collaborative behavior. Moreover, such systems are often user centric excluding the context around them. For instance, many wrist worn devices are able to monitor the heart rate of a user. However, the heart rate relates heavily to the activity a user is currently performing, which also needs to be considered by the monitoring device. Modeling such activity as a whole would involve a multitude of sensors which need to communicate efficiently with each other and which need to collaborate autonomously with each other in order to optimize their own goals and performance and to provide relevant information to other components. Such a complete model of ambient intelligence would be able to comprehend the underlying environment at various degrees of realism including the devices therein, the interaction between them and of course the users that utilise them. Moreover, it would need to be adaptive itself promoting the concept of self-evolution by which systems and services, extend, adapt and optimise themselves in dependence of the underlying environment and the use. Based on such a model of self-awareness, cognitive intelligence would be able to sense, anticipate, adapt and, eventually, optimise the perceived context and behaviour autonomously and as such would be able to adapt to different, potentially unknown, situations without the need for any user intervention or configuration [5]. Nevertheless, how such intelligence is realised, where it is facilitated and how it is engineered within complex, heterogeneous and highly dynamic infrastructures is very much subject to current and future research. As discussed in [6], there is a need to embed a high degree of self-awareness into virtual any component and service of an intelligent environment, which would reflect a distributed sensor model as discussed, e.g., in [9] where raw data is processed locally by sensors (or smart components) themselves before more expressive, relevant knowledge is propagated into the environment. Such a sensing model would not only address scalability issues by decomposing computational requirements but would also provide for a more globally oriented model of self-awareness to emerge autonomously in a bottom-up fashion as discussed in [6]. If such sensors provide sufficient processing power then they could form the layer required upon which cognitive intelligence could be facilitated. Clark et al. have discussed such a knowledge-based middle layer conceptually in the context of the Internet of Things [7], and a similar concept has been proposed in [8] for sensor-rich smart environments. In both cases it is argued that raw data, from a conceptually lower oriented physical layer, needs to be modelled, analysed and enriched in order to provide well structured, understandable and, eventually, useful knowledge to users and services that operate on a, conceptually, higher oriented application layer. Moreover, it is argued that such a layer needs to be closely interwoven with the environment itself and with the services that operate on them. This, in particular would support the concept of self-evolution in a way that the environment would shape the services that operate therein and, vice versa, the services would influence the environment they operate on. This is similar to the concept proposed by Bionets (www.bionets.eu) where biological inspired concepts adapt autonomously thus blending the network and its services into a single evolving entity. Nevertheless, how such a layer can be realised, the physical components it is based on or the interfaces and algorithms required for a coherent self-adapting model is yet unclear. Furthermore, such a layer would also need to provide actionable interfaces to the underlying components on which distinct adaptive measures could be executed upon. This aspect in particular is necessary to make the transition from context aware to pro-active and responsive environments as required by ambient intelligence [4].

The aim of the related Disappearing Computer initiative is to "see how information technology can be diffused into everyday objects and settings, and to see how this can lead to new ways of supporting and enhancing people's lives that go above and beyond what is possible with the computer today" (www.disappearing-computer.net). The basic foundations of such a framework in which everyday objects are augmented with relevant sensing and control capabilities have been explored by a number of research initiatives such as the Smart-Its project (www.smart-its.org), which investigated the concept of smart artefacts by utilizing them as generic building blocks for ubiquitous computing scenarios. The aim of this project was to augment every day objects with various sensing, processing and communication functionality to research contextual awareness and emerging behaviour among collections of objects. Similarly, the Haggle (www.cl.cam.ac.uk/research/srg/netos/haggle/) project has researched opportunistic behavior in the context of future oriented networking and communication paradigms. The SAPERE (www.sapere-project.eu) project aims at modeling digital infrastructures, its devices, information services and 
users, through a 'distributed computational ecosystem' that is inspired by natural principles and which laws are enforced by services that adapt in dependence of the underlying environment. The ASCENS (www.ascens-ist.eu) project proposed a system where service-component ensembles are dynamically composed to form hierarchical oriented compositions that are connected through a highly dynamic and volatile infrastructure and which promote future autonomic and self-aware systems.

\section{REQUIREMENTS}

For future cognitive sensor networks to become a reality, virtually every concept, sensor or sub-network therein has to become intelligent itself in dependence of their individual properties. The aim of which is to provide each component with a high degree of understanding of its own operational status, its context of use as well as its interrelation with other components and the users that are available within the same environment and, in some cases, beyond. This relates to the problem of translating sensed or other contextual information into sensor or sensor network specific profiles that can be processed autonomously. Nevertheless, the usefulness of such an environment would still be very limited if such sensors would only exit in isolation. That is, if they would not communicate with other sensors or sensor clusters about their current context of use or about their collective use as required for the modeling of more complex tasks and behavior. This in particular, emphasizes the need for a conceptual layer that connects to the lower oriented physical world (represented by the collection of sensors) and which self-organizes the available information as well as the sensors therein based on their properties or context of use and which also promotes the communication and interaction between individual sensors and the network as a whole. In essence, such a layer would reflect a networked intelligence that would bridge the gap between the isolated use of components and complex and coordinated services, which would provide the platform on which a layer of cognitive intelligence can emerge. Thus initial requirements for the emergence of cognitive behavior can be summarized as follows:

- Facilitating self-awareness at sensor level as well as on concept level. The latter of which may involve multiple sensors or sensor clusters as required to model more complex behavior.

- Self-organizing knowledge structures that are able to reflect an up-to-date model of the underlying environment and which can be used for real-time decision making. Here, the multi-dimensional space of the domain concerned and its spatial, social and business related aspect is of particular interest.

- Collaborative behavior among components, which would also involve the dynamic orchestration of sensors or sensor clusters in order to model dependent behavior. For instance, a heart rate monitor would need to collaborate with an activity monitor to correctly assess the sensed heart rate.

Other relevant requirements, which are discussed next, are based non-deterministic and opportunistic character of future systems and by the necessity for predictive capabilities.
Current design principles are often based on deterministic behavior, which, although a requirement for many computational systems, is no longer viable for future heterogeneous sensor-based systems that face a high degree of uncertainty in, e.g., the availability of resources, the correctness of information, changing goals and parameters. Consequently, opportunistic behavior will play a pivotal role for the realization and the efficiency of future cognitive systems where many tasks need to be performed not when desired but whenever possible. The free Dictionary (www.thefreedictionary.com) defines opportunism as the "conscious policy and practice of taking selfish advantage of circumstances, with little regard for principle". In other words, opportunistic behavior partially supports the 'survival of the fittest' principle, which is a cornerstone for self-evolving systems and mechanisms. Thus, principles to support opportunistic behavior in relation to the availability of resources, the non-sequential execution of service composition and for the promotion of collaborative behavior need to be incorporated into such systems.

Similar, the concept of emerging behavior needs to be integrated into such systems in order to deal with their dynamics. In this context, emerging behavior needs to be based on the sensors themselves and on the communication and interaction between them. This in particular reflects a type of networked intelligence that is not just embedded into the underlying infrastructure but actually based on their use and augmented with the information therein. This would allow for services to be autonomously composed and configured in dependence of the goals they need to serve. Moreover, they would be adaptive in dependence of the dynamicity of the network and would be more resilient with respect to the availability of resources. This reflects a bottom-up approach of evolutionary computing that offers new perspectives on the facilitation of such networked and highly distributed yet collaborative intelligence. In particular, the requirements for the autonomous and spontaneous collaboration of sensors in support for specific tasks and the autonomous composition and configuration thereof need to be further investigated. Bio-inspired mechanisms such as stigmergy, swarm intelligence and generic fitness measures may prove to be a good starting point to promote and evaluate collective behavior, which will in turn provide the stimulus for emerging behavior. The resulting intelligence may then be able pervade the underlying infrastructure and its components and will thus reduce the barrier between the physical environment and the services that operate on them.

Another characteristic relevant for cognitive systems is that of prediction, which could lead towards proactive components and interfaces that are adaptive and anticipatory in nature. Such mechanisms would provide the ability to anticipate possible future information, situations or even interaction between components within loosely coupled networked systems. This means that, based on the observation and analysis of past behavior and the use of predictive reasoning, individual sensors could predict their own future states for various aspects of, e.g., their own operational environment or their interrelations with other components to either guide themselves to a more optimum state or, if necessary, to prevent unwanted or dangerous situations before their actually occur. Such a model 
of temporal self-awareness represents a significant research challenge, which will require some sort of temporal overlays that are capable to differentiate between past, current and future system conditions. The incorporation thereof would provide the knowledge base for flexible cognitive mechanisms that are anticipatory in nature and which would have the ability to adapt to new, previously unknown conditions and situations, which is a fundamental requirement for cognitive intelligence.

Future large-scale sensor based environments will be characterized by large amounts of information that will be available from a multitude of sources to be used for various purposes. For them to be useful, they need to be properly organized and enriched and they need to be accessible in real-time to other services in order to e.g. compute and execute adaptive measures in time. For that it will be necessary to self-organize available information along various dimensions. Moreover, such knowledge needs to be organized not only along individual dimensions but also across them thus constructing multiple interrelated overlay networks that reflect technical, social as well as domain specific dimensions and the relation between them. Furthermore, the organized knowledge needs to be constantly analyzed to pre-compute aggregates, to extract knowledge at higher granular levels and to cluster sensors based on their self-aware profiles, which could be the stimulus for collaborative behavior. The resulting knowledge ecosystem will be a reflection of the participants of the environment, their operational status and interactions as well as their context of use, which would be available to all components of the network.

Latest advances in sensor and networking technologies has led to a number of platforms for wireless sensor networks that provide unique opportunities to perceive the environment, facilitate multi-modal sensor processing, to communicate with other self-similar sensor components of the environment in order to achieve a general understanding thereof and to actuate mechanisms [10]. They provide the level of abstraction required to deal with the heterogeneous nature of pervasive environments. Nevertheless, as discussed in [11], it is not just the quantity of sensors or sensed information that is important but it is the contextual relations between them and the interpretation thereof. Thus, for useful applications to emerge it is necessary to self-organize sensors and the networks thereof in relation to their contextual proximity. Expressing and understanding contextual proximity however requires a cognitive intelligence that is capable to comprehend all aspects of the underlying environment including the components and the users therein the interaction between them and the knowledge available through them.

Although the possibilities for individual applications and services in the area of pervasive healthcare and beyond are practically limitless, their success will ultimately depend on the cost-effectiveness of all aspects of the technology involved, which is particularly relevant for volatile or disposable items for which associated costs need to be well below the production or retail costs thereof. Equally important, configuration, maintenance and service deployment will play a vital role and if not automated may prove fatal for future sensor based systems. Consequently, cognitive and self-adapting principles that allow for emerging behaviour will be paramount for future intelligent environments and the sensor networks therein.

\section{CONCLUSIONS AND FUTURE WORK}

This paper discussed the potential and some of the requirements of future sensor based environments for them to become more intelligent and, ultimately, cognitive-aware. Such awareness would not only lead to more autonomous systems, applications and services but would also pave the way for new types of human-environment interactions, which are based directly on the information and interaction perceived rather than on the, often error prone, information manually provided. In turn, this has the potential for the provision of new types of monitoring, assistive and even enabling services in the area of pervasive healthcare and beyond.

Future work will therefore need to concentrate on the modelling of situations as well as activities, the communication between individual sensor nodes and virtual, purpose-based, node clusters as well as the efficient and fully distributed analysis of the perceived environment with the goal to compute and execute measures that are somehow advantageous to the purpose of the underlying environment or the activities and interactions therein.

\section{REFERENCES}

[1] Aleksandar Milenkovic, Chris Otto, and Emil Jovanov; "Wireless sensor networks for personal health monitoring: Issues and an implementation", Comput. Commun. 29, 2521-2533, pp13-14, 2006

[2] Andreas Zinnen, Ulf Blanke, and Bernt Schiele; "An Analysis of SensorOriented vs. Model-Based Activity Recognition" In Proceedings of the 2009 International Symposium on Wearable Computers (ISWC '09). IEEE Computer Society, Washington, USA , pp 93 - 100, 2009

[3] Shumei Zhang, Paul McCullagh, Chris Nugent, Huiru Zheng, Matthias Baumgarten, "Optimal model selection for posture recognition in homebased healthcare", International Journal of Machine Learning and Cybernetics, DOI 10.1007/s13042-010-0009-5, Springer Verlag, 2010

[4] Diane J. Cook, Juan C. Augusto, and Vikramaditya R. Jakkula. 2009. Review: Ambient intelligence: Technologies, applications, and opportunities. Pervasive Mobile Computing, pp 277 - 298, 2009

[5] G. Michael Youngblood, Diane J. Cook, and Lawrence B. Holder. 2005. A learning architecture for automating the intelligent environment. In Proceedings of the 17th conference on Innovative applications of artificial intelligence - Volume 3 (IAAI'05), Bruce Porter (Ed.), Vol. 3. AAAI Press 1576-1581.

[6] Baumgarten, M., Guldenring, D., Poland, M. P., Nugent, C., Hallberg, J.; "Embedding Self-Awareness into Objects of Daily Life - The Smart Kettle", Proceedings of the 6th International Conference on Intelligent Environments, DOI 10.1109/IE.2010.50, pp. 34 - 39, 2010

[7] David D. Clark, Craig Partridge, J. Christopher Ramming, and John T. Wroclawski. 2003. A knowledge plane for the internet. In Proceedings of the 2003 conference on Applications, technologies, architectures, and protocols for computer communications. ACM, USA, pp 3-10. DOI $=10.1145 / 863955.863957$

[8] Baumgarten, M., Bicocchi, N., Curran, K., Mamei, M., Mulvenna, M.D., Nugent, C., Zambonelli, F.; "Towards Self-Organizing Knowledge Networks for Smart World Infrastructures", International Transactions on Systems Science and Applications (ITSSA), Vol. 1, No. 3/4, ISSN 1751-1461, pp 123 - 133, 2006

[9] Akyildiz, I.F., W. Su, Y. Sankarasubramaniam, E. Cayirci, "A Survey on Sensor Networks", IEEE Communications Magazine, August, 102114(2002) (IEEE Communications Society Best Tutorial Paper Award)

[10] Pauwels E., Salah A.A., Tavenard R.; "Sensor Networks for Ambient Intelligence," Workshop on Multimedia Signal Processing, 2007

[11] Holmquist L.E., Mattern F., Schiele B., Alahuhta P., Beigl M., Gellersen, H.W.; "Smart-Its Friends: A Technique for Users to Easily Establish Connections between Smart Artefacts", 3rd Int. Conf. on Ubiquitous Computing, Atlanta, GA, USA, 2001 